\title{
Phytoprotection
}

\section{Index des sujets, volume 80 Subject Index, Volume 80}

Volume 80, numéro 3, 1999

URI : https://id.erudit.org/iderudit/706194ar

DOI : https://doi.org/10.7202/706194ar

Aller au sommaire du numéro

Éditeur(s)

Société de protection des plantes du Québec (SPPQ)l

ISSN

0031-9511 (imprimé)

1710-1603 (numérique)

Découvrir la revue

Citer ce document

(1999). Index des sujets, volume 80. Phytoprotection, 80(3), 197-199.

https://doi.org/10.7202/706194ar d'utilisation que vous pouvez consulter en ligne.

https://apropos.erudit.org/fr/usagers/politique-dutilisation/ 


\section{Index des sujets, volume 80 Subject Index, Volume 80}

\section{A}

Abies balsamea

Acer spp.

Acrobasis vaccinii

Aedes triseriatus

Agrostis palustris

Algérie/Algeria

Allium cepa

Anagyrus kamali

Anoplophora glabripennis

Aphidius nigripes

arrhénotoquie/arrhenotoky

Avena sativa

Azadirachta indica

B

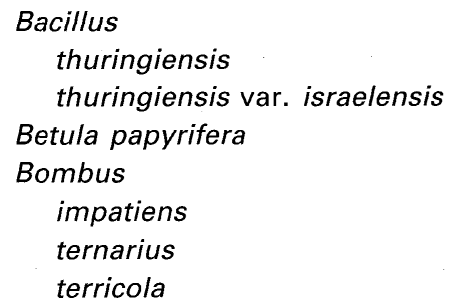

Botryodiplodia hypodermia

Botrytis squamosa

Brassica

napus

napus var. oleifera oleracea var. capitata oleracea var. italica rapa

\section{C}

Caliciopsis pinea

Callosobruchus maculatus

Campylomma verbasci

Caraïbes/Caribbean

Carex spp.

Cercospora carotae

chitosane

Choristoneura rosaceana

Chrysoperla rufilabris
Clavibacter michiganensis subsp. michiganensis 115 michiganensis subsp. sepedonicus 38

Coccinella septempunctata 38

Coleomegilla maculata lengi 38

collembole/collembola 191

Conotrachelus nenuphar 193

Cronartium ribicola 44

Cryptolaemus montrouzieri 50, 103

cultures transgéniques 52,71

Cylindrocarpon destructans 44

floridanum 44

\section{D-G}

Daucus carota 37, 41

Dothiorella ulmi 37

entomofaune/entomofauna 187, 189

Erwinia carotovora $\quad 41$

Fusarium

graminearum 47,48

oxysporum 44

187 oxysporum f.sp. radicis-lycopersici 137

187 Galapagos 187

187

Glomus

etunicatum 41

intraradices 41

Glycine max $\quad 45,49,52,71$

52,71 Gossypium hirsutum 52,71

Gremmeniella abietina $\quad 55$

39,192

39,192

71

\section{H-L}

Harmonia axyridis $\quad 38$

Helminthosporium solani 42

Heterorhabditis bacteriophora 190

44 Hippodamia tredecimpunctata tibialis 38

42 Hordeum vulgare 36, 169

193 induced resistance 137

50, 103

185

37

137

Inonotus

circinatus

39

tomentosus $\quad 39$

insolation hivernale $\quad 46$

49, 51 laboratoire de diagnostic 43, 115

$186 \quad$ Lactuca sativa 50, 51, 121 
Lambdina fiscellaria

Leiodidae

Leptosphaeria maculans

Lotus corniculatus

Lycopersicon esculentum $36,40,52,71,85$

Lymantria dispar

\section{$\mathbf{M}-\mathbf{O}$}

Maconellicoccus hirsutus

Macrosiphum euphorbiae

Malus pumila

Manduca sexta

Meloidogyne hapla

modélisation/modelling

mycobiote/mycobiota

nématodes/nematodes

Nicotiana tabacum

Ocimum

basilicum

gratissimum

suave

Ostrinia nubilalis

\section{P-R}

paclobutrazol

Phellinus pini

phéromone/pheromone

Phytophthora

fragariae

infestans

phytotoxine/phytotoxin

Picea

abies

mariana

spp.

Pieris rapae

Pinus

banksiana

pinaster

resinosa

strobus

sylvestris

spp.

Pissodes strobi

Poa annua

Polistes versicolor

pollinisation/pollination

Populus spp.

Pratylenchus penetrans

Propylea quatuordecimpunctata
186, 191

185

50,103

186

21, 193

188

36

193

1

36

52,71

42

42

42

190, 192

65

39

186, 192

47

$40,41,85$

45

187

44

39,187

39,192

\section{5}

44

55

44

35

97, 187

187

65

187

185

46

179

38
Prunus serotina

190

Pseudomonas marginalis

38

Psithyrus spp.

187

ré-émergence/re-emergence 43, 48, 115, 127

résistance induite

137

Rhagoletis pomonella

21

Rhizoctonia solani

45

Rhizotrogus majalis

190

Rhopalosiphum

maidis

169

padi

Rubus

chamaemorus

idaeus

169

185

47

S

Schizaphis graminum $\quad 169$

Sclerotinia

homocarpa

sclerotiorum

Scymnus coccivora

$41,45,49$

Sitobion fragariae

50,103

169

Sitodiplosis mosellana $\quad 46$

Sitophilus oryzae $\quad 190$

Smicronyx spp.

48

Solanum tuberosum

$38,40,41$,

$42,43,45,71,85$

Sorghum bicolor

190

Steinernema spp.

190

Streptomyces

hygroscopicus var. geldanus

47 scabies

45

Striga hermonthica

48

\section{T-U}

tébuconazole

48

Thanathephorus cucumeris $\quad 45$

thaxtomine A/thaxtomin A 45

thélytoquie/thelytoky $\quad 192$

Tomicus piniperda 35, 97

transgenic crops $\quad 52,71$

Trichoderma spp. 1, 137

Trichogramma

pretiosum 39, 192

spp. 192

Trichoplusia ni 39, 192

Trifolium pratense 36

Triticum aestivum 13, 46, 47, 48, 127, 169

Ulmus americana 37

Ustilago tritici 13 


\section{V-Z}

Vaccinium oxycoccos

Vigna unguiculata

Virus

BYDV

INSV

PVX

PVY

SNPV

TSWV

winter insulation

Xanthomonas

campestris $p v$. vitians

hortorum pv. vitians

spp.

Zea mays
186

42

169

115

43, 115

43

49

115

46

51, 121

50

137

$45,52,71,189$ 